\title{
Expression of the 17-1A antigen in gastric and gastro-oesophageal junction adenocarcinomas: a potential immunotherapeutic target?
}

\author{
Iain G Martin, Simon G Cutts, Kevin Birbeck, Sally Gray, Philip Quirke
}

\begin{abstract}
Background-A murine monoclonal antibody against the 17-1A epithelial antigen has been shown to be a useful adjuvant therapy in colorectal cancer. Its clinical use could be extended to patients with upper gastrointestinal adenocarcinoma. Aim-To determine the distribution of the antigen in gastric and oesophageal adenocarcinoma.

Methods-The activity of two monoclonal antibodies active against 17-1A epithelial antigen was studied in gastric and gastrooesophageal junction adenocarcinomas: fresh frozen tissue from both the carcinoma and adjacent mucosa was stained using immunocytochemistry with a murine monoclonal antibody (17-1A edrecolomab ${ }^{\circledR}$, Glaxo Wellcome); paraffin embedded tissue was stained using the humanised monoclonal antibody 3622 W94 (Glaxo Wellcome).
\end{abstract}

Results-29 of 33 cancers ( $88 \%)$ stained with the murine antibody and 39 of 40 $(98 \%)$ with the humanised antibody. The degree of staining was greater in well differentiated and moderately differentiated tumours. There was no staining of the normal background gastric or oesophageal mucosa, but areas of intestinal metaplasia stained intensely. The humanised monoclonal 3622W94 antibody produced more intense staining than the murine antibody.

Conclusions-The high incidence of expression of the 17-1A antigen in patients with gastric and gastro-oesophageal junction adenocarcinomas suggests a potential role for these antibodies as an adjuvant treatment for these common cancers.

(F Clin Pathol 1999;52:701-704)

Surgery, The Centre for Digestive Diseases,

The General Infirmary at Leeds

I G Martin

Division of Molecular Oncology, University of Leeds

K Birbeck

S Gray

Correspondence to: Mr I G Martin, Academic Department of Surgery, The General Infirmary at Leeds, Great George Street, Leeds LS1 3EX, UK.

email i.g.martin@leeds.ac.uk

Accepted for publication 14 May 1999

neoplasms; immunotherapy

Carcinoma of the stomach remains one of the most common human cancers worldwide. In the United Kingdom, there are 10-12 000 new cases a year. While with early diagnosis and radical surgery, some progress has been made in improving the outlook for patients with this disease, the overall five year survival is little better than $20 \%{ }^{1}$ For the $6-7000$ patients who present each year in the United Kingdom with oesophageal cancer the outlook is bleaker still, with only $4-5 \%$ surviving to five years. ${ }^{2}$ Although the numbers of patients with oesophageal cancer is smaller, the incidence of adenocarcinoma of the distal oesophagus is rising by approximately $10 \%$ per annum in the Western world. ${ }^{3}$ A large number of patients present with disease which, although amenable to surgical resection, is subject to a significant chance of recurrence. Traditional adjuvant chemotherapy seems to have little to offer the patient with gastro-oesophageal adenocarcinoma. In Japan adjuvant immunotherapy with non-specific agents such as the protein bound polysaccharide PSK $^{4}$ have been shown to be of benefit in a randomised controlled trial, increasing five year survival from $60 \%$ to $73 \%$ in patients with gastric cancer undergoing potentially curative resection, but such treatment is not available outside of Japan and Korea. Recently the use of a murine monoclonal antibody to the antigen 17-1A (murine monoclonal antibody 17-1A edrecolomab ${ }^{\circledR}$, Glaxo Wellcome) has been shown to produce a $30 \%$ reduction in the risk of death when used in the adjuvant setting in patients with colorectal cancer. ${ }^{5}$ The humanised monoclonal antibody 3622W94 (Glaxo Wellcome) is active against the same antigen but has shown greater affinity than the murine antibody; however, its use in clinical trials has not yet been reported.

It seemed to us a logical step to consider the potential use of similar therapeutic strategies in patients with gastric and oesophageal adenocarcinoma. Although the staining of gastric cancers with the murine 17-1A monoclonal antibody has been reported in a small number of patients, ${ }^{6}$ this was felt to be insufficient evidence on which to undertake a clinical trial. A study was conducted to ascertain the staining pattern of normal gastric and oesophageal mucosa and gastro-oesophageal adenocarcinomas; if a significant proportion of cancers stain with antibodies to the 17-1A antigen then clinical trials of such agents in patients with gastric and oesophageal adenocarcinoma may be considered.

\section{Methods}

TISSUE STAINING WITH 17-1A EDRECOLOMAB Fresh, snap frozen tissue was studied from 33 patients with gastric and gastro-oesophageal junction carcinoma. There were 18 tumours of the upper stomach and gastro-oesophageal junction, seven of the middle third of the stomach, and eight of the distal third of the stomach. These were selected at random from our store of frozen tissue held within the department of pathology.

There were 28 male and five female patients. Their median age was 69 years (range 44 to 87). There were five UICC TNM stage I 
tumours, four stage II, 17 stage III, and seven stage IV. Three of the tumours were well differentiated, 12 moderately differentiated, and 18 poorly differentiated.

As an internal control, five cases of normal colonic mucosa and five of colonic cancer were also stained. These were studied as the expected staining pattern is well described. ${ }^{6}$

Using both normal and tumour tissue, three $6 \mu \mathrm{m}$ cryostat sections were cut and dried thoroughly. After fixation in acetone for 10 minutes at room temperature, one section was incubated with the antibody $(5 \mathrm{mg} / \mathrm{ml})$ for one hour. The second section was used as a negative control with the omission of the antibody. The sections were washed in phosphate buffered saline (PBS) and incubated with biotin for 30 minutes, then washed again with PBS and incubated with streptavidin-biotin complex for 30 minutes (Duet kit, Dako). After washing, the sections were incubated for 20 minutes in $0.15 \%$ diaminobenzidine (DAB) containing $0.02 \%$ hydrogen peroxide and counterstained with haematoxylin. The third section was stained with haematoxylin and eosin for the histological assessment of "normal" tissue and to confirm the presence and type of tumour.

We were unable to obtain specific staining of paraffin embedded tissue with this antibody (data not shown), even with microwave enhancement; this was not surprising given that other workers have been unable to achieve reliable staining in paraffin embedded tissues. It seems probable that the antigen retrieval methods used on the paraffin sections do not produce sufficient exposed antigen for the weaker affinity murine antibody to give significant staining.

TISSUE STAINING WITH 3622W94

Paraffin embedded archive material from 40 patients undergoing gastrectomy or oesophagogastrectomy for adenocarcinoma of the stomach or gastro-oesophageal junction was studied. There were 14 tumours arising in distal third of the stomach, 14 from the middle third of the stomach, and 12 from the proximal third and gastro-oesophageal junction. There were 30

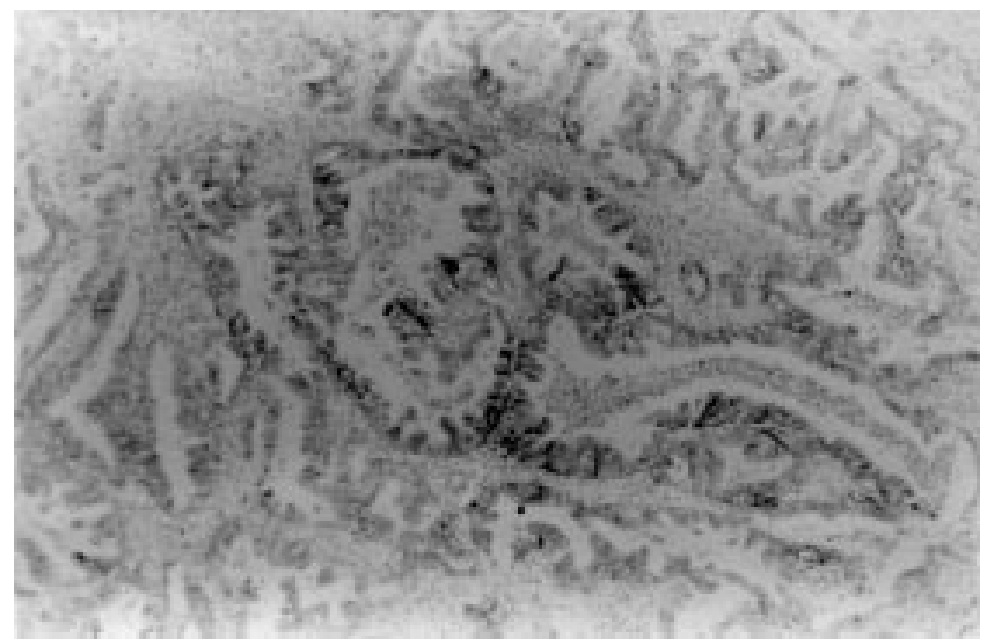

Figure 1 Moderate staining of a gastric carcinoma with the murine monoclonal antibody. male patients and 10 female patients. Their median age was 73 years (range 45 to 87 ). There were six patients with UICC TNM stage I disease, seven with stage II, 20 with stage III, and seven with stage IV. Three of the tumours were well differentiated, 17 moderately differentiated, and 20 poorly differentiated.

In addition, paraffin embedded archive material from 10 patients with carcinoma of the colon was studied as an internal control.

Three $4 \mu \mathrm{m}$ sections were cut and placed on slides. The sections were dewaxed and taken through three changes of absolute alcohol. Endogenous peroxidase activity was blocked using a $2 \%$ solution of hydrogen peroxide in methanol. After washing in water the sections were placed in trypsin solution for one minute and then in a microwave oven for six minutes. The slides were washed and blocked with protein block serum free (Dako X0909) and Vector avidin/biotin blocking kit (Vector SP2001). The sections were incubated in fluorescein conjugated 3622W94 antibody diluted $1: 100$ for one hour and subsequently in biotinylated antifluorescein (Vector BAO6O1). Sections were then incubated in a streptavidinbiotin complex (Vector PK6102). The slides were developed in diaminobenzidine solution and incubated in copper sulphate solution. Finally the slides were counterstained with haematoxylin, dehydrated, cleared, and mounted in a synthetic mounting medium.

\section{ASSESSMENT OF GRADING}

Each section was reviewed by a single pathologist and the staining with either 17-1A edrecolomab or 3622W94 was graded using an empirical semiquantitative system. The staining was membranous in all cases: -, negative; + , weak staining throughout the tumour or patchy moderate staining; ++ , moderate staining throughout or patchy intense staining with weaker areas; +++ , strong staining throughout the tumour.

\section{Results}

MURINE ANTIBODY 17-1A EDRECOLOMAB

The pattern seen in the colonic mucosa and colonic carcinomas was as expected, with all the sections staining. The normal mucosa stained strongly in all cases and the colonic tumours stained moderately in four cases and weakly in one. In neither the stomach nor the oesophagus did the normal mucosa stain with the antibody in any case.

Of the 33 tumours studied, 10 stained strongly, nine moderately, and 10 weakly. Thus overall 29 of the 33 cases of gastro-oesophageal cancer $(88 \%)$ stained with the murine antibody to $17-1 \mathrm{~A}$ (fig 1 ).

Of the 15 well or moderately differentiated tumours, one did not stain, three stained weakly, four moderately, and seven strongly. In the 18 patients with poorly differentiated tumours, three did not stain, seven stained weakly, five moderately, and three strongly. Although there was a tendency for the better differentiated tumours to stain more intensely (table 1$)$ this was not significant $\left(p=0.5, \chi^{2}\right.$ test). 
Table 1 Patterns of staining with the two antibodies in gastric and gastro-oesophageal junction tumours

\begin{tabular}{lll}
\hline & $\begin{array}{l}\text { Murine antibody, 17-1A } \\
\text { edrecolomab }\end{array}$ & $\begin{array}{l}\text { Humanised monoclonal } \\
\text { antibody 3622W94 }\end{array}$ \\
\hline All tumours & $88 \%(29 / 33)$ & $98 \%(39 / 40)$ \\
- & $(4)$ & $(1)$ \\
+ & $(10)$ & $(1)$ \\
++ & $(9)$ & $(15)$ \\
+++ & $(10)$ & $100 \%(12 / 12)$ \\
$\begin{array}{l}\text { Gastro-oesophageal junction and } \\
\text { proximal third of stomach }\end{array}$ & $83 \%(15 / 18)$ & $93 \%(13 / 14)$ \\
$\begin{array}{l}\text { Middle third tumours } \\
\text { Distal third tumours } \\
\text { Well / moderately differentiated } \\
\text { tumours }\end{array}$ & $100 \%(7 / 7)$ & $100 \%(14 / 14)$ \\
Poorly differentiated tumours & $88 \%(7 / 8)$ & $100 \%(20 / 20)$ \\
\hline
\end{tabular}

Values in parentheses are the numbers of patients staining.

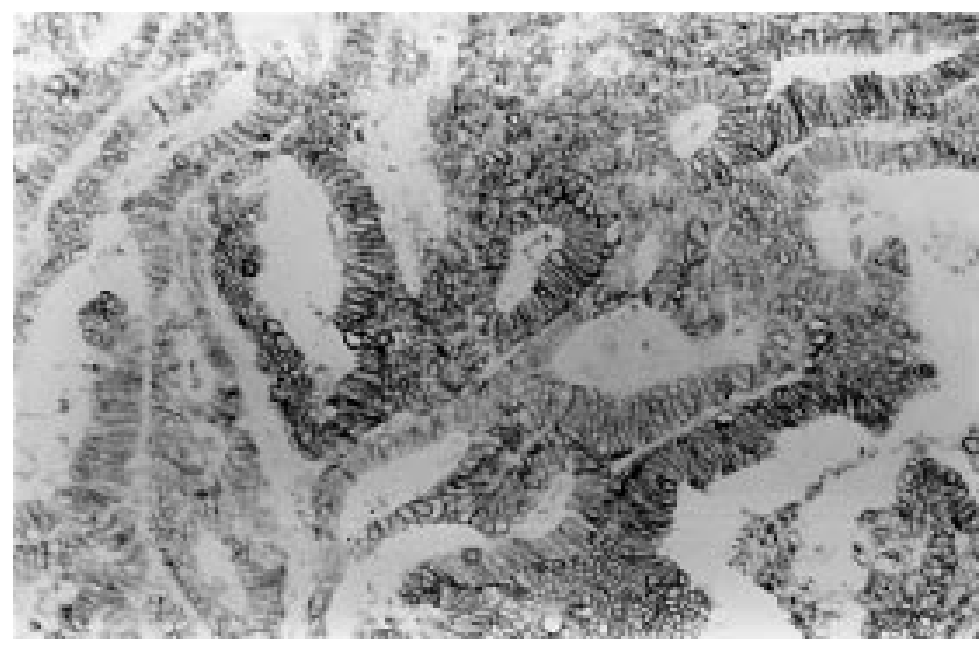

Figure 2 Strong staining of a gastric carcinoma with the 3622 W94 humanised antibody.

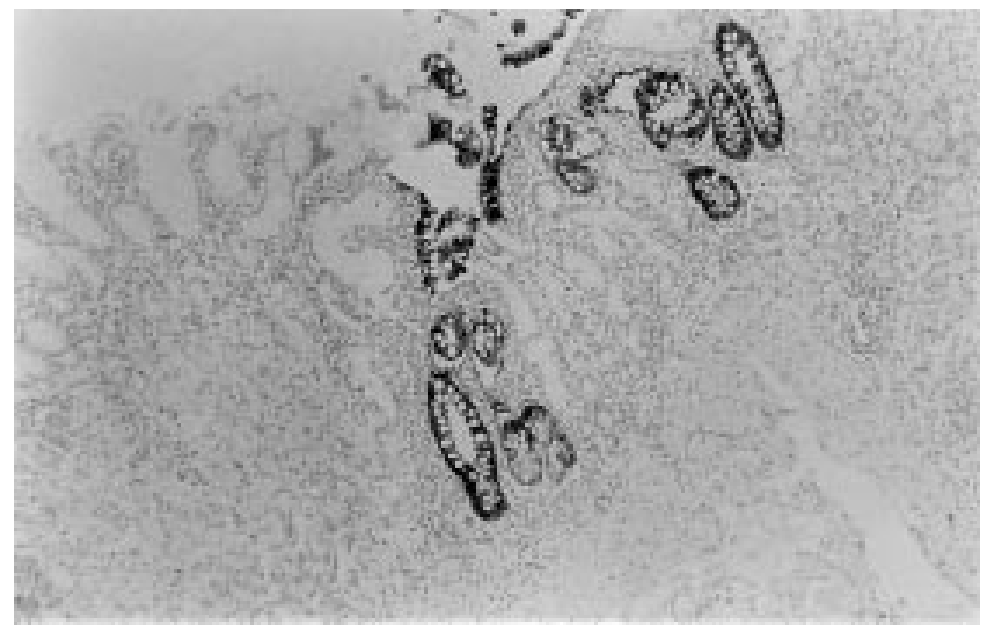

Figure 3 Strong staining of a patch of intestinal metaplasia within a field of "normal" gastric mucosa (3622W94 humanised antibody).

There was no staining of normal gastric mucosa in any of the cases examined. Although not part of the protocol, it was interesting to observe that in all of the 12 cases which contained patches of intestinal metaplasia within the "normal" gastric mucosa, the intestinal metaplasia stained strongly with the antibody.

HUMANISED MONOCLONAL ANTIBODY 3622W94

All the 10 cases of colonic carcinoma used as controls stained strongly with 3622 W94, both in the tumour tissue and in the normal colonic mucosa. The normal gastric or oesophageal mucosa did not stain at all in 36 of the 40 cases, just four cases showing occasional very weak and patchy staining.

Of the 40 gastric and gastro-oesophageal junction carcinomas, 39 (98\%) stained with 3622 W94 (fig 2). Staining in 23 of the cases was strong, in 15 moderate, and in just one case weak. There was no relation between the intensity of staining and the degree of differentiation of the tumour, the location of the tumour, or the stage of the tumour (table 1). As with the murine antibody, patches of intestinal metaplasia always stained intensely $(++/+++)$ (fig 3$)$.

\section{Discussion}

The $17-1 \mathrm{~A}$ antigen is a $37 \mathrm{kDa}$ protein expressed on the cell surface across a wide variety of human epithelia. ${ }^{6} \mathrm{~A}$ murine antibody to this antigen has shown potential as an adjuvant treatment for patients with Dukes grade C colorectal cancer. ${ }^{58}$ The potential benefit of this treatment is considerable, with a $32 \%$ reduction in mortality at seven years follow up in patients with Dukes grade C colorectal cancer. ${ }^{8}$ Gastric cancers have previously been shown to stain, ${ }^{6}$ but that report contained information on only nine cases and the finding was not the main focus of the report. Few if any data are available on patients with oesophageal adenocarcinoma.

As far as we are aware this is the first series to study a significant number of patients with gastro-oesophageal cancer. We have shown that gastric and gastro-oesophageal junction adenocarcinomas show specific immunoreactivity to the murine monoclonal antibody 17-1A edrecolomab, with nearly $90 \%$ of the cancers examined staining. The level and intensity of staining was greater with the humanised monoclonal antibody 3622W94, with $98 \%$ of the carcinomas staining. This high level of staining suggests that adjuvant immunotherapy with monoclonal antibodies may have a role in the treatment of these two common cancers. There is evidence that immunotherapy has a role in the adjuvant treatment of gastric cancer, ${ }^{4}$ but the agents used are currently not available for use outside Japan and Korea. We believe the potential availability of a relatively non-toxic immunological adjuvant warrants further exploration in upper gastrointestinal cancer.

These two antibodies do not stain normal gastric or oesophageal mucosa but-perhaps as would be expected-both stain patches of intestinal metaplasia strongly. Although the stratified squamous epithelium of the oesophagus does not stain, it has been observed previously that the epithelium of the normal mucus secreting oesophageal glands does stain. ${ }^{7}$ The degree of staining of the tumours with the murine monoclonal antibody was related to degree of differentiation. However, with the humanised antibody 3622W94, tumours of all degrees of differentiation stained similarly.

This small study provides us with the background information required to begin to develop possible strategies for the use of monoclonal antibodies directed against this 
antigen in the adjuvant treatment of patients with gastric and possibly oesophageal adenocarcinoma. Given the poor response of both of these cancers to conventional adjuvant chemotherapy, the use of this novel immunotherapy warrants clinical study in patients with gastrooesophageal carcinoma.

1 Forman D, Rider L, eds. Stomach cancer. In: Cancer in Yorkshire: cancer registry report 1996. Leeds: Leeds Yorkshire: cancer registry

2 May J, Melling P, Brown J, et al. Oesophageal cancer in Yorkshire: a regional audit. Gut 1994;35:S14.

3 Blot WJ, Devesa SS, Kneller RW, et al. Rising incidence of adenocarcinoma of the esophagus and gastric cardia.
ade fAMA 1991;265:1287-9.
4 Nakazato H, Koike A, Saji S, et al. Efficacy of immunochemotherapy as adjuvant treatment after curative resection of gastric cancer. Study Group of Immunochemotherapy with PSK for Gastric Cancer. Lancet 1994;343:1122-6.

5 Riethmuller G, Schneider-Gadicke E, Schlimok G, et al. Randomised trial of monoclonal antibody for adjuvant therapy of resected Dukes' C colorectal carcinoma. Lancet 1994;343:1177-83.

6 Gottlinger HG, Funke I, Johnson JP, et al. The epithelial cell surface antigen $17-1 \mathrm{~A}$, a target for antibody-mediated tumour therapy: its biochemical nature, tissue distribution and recognition by different monoclonal antibodies. Int $\mathcal{F}$ Cancer 1986;38:47-53.

7 GlaxoWellcome Research and Development. PanorexInvestigators Brochure. Uxbridge: Glaxo Wellcome, 1996:5.

8 Riethmuller G, Holz E, Schlimok G, et al. Monoclonal antibody therapy for resected Dukes $\mathrm{C}$ colorectal cancer: seven year outcome of a multicenter randomised trial. I Clin Oncol 1998;16:1788-94. 